\title{
Preparation of lampbrush chromosomes dissected from avian and reptilian growing oocytes
}

\author{
Alsu Saifitdinova1, Svetlana Galkina², \\ Valeriia Volodkina ${ }^{3}$, and Elena Gaginskaya ${ }^{3}$ \\ ${ }^{1}$ Chromas Research Resource Center of Saint Petersburg State University Research Park, \\ Oranienbaumskoe sh. 2, Stary Peterhof, Saint Petersburg, 198504, Russian Federation \\ 2 Department of Genetics and Biotechnology, Saint Petersburg State University, \\ Universitetskaya nab. 7-9, Saint Petersburg, 199034, Russian Federation \\ 3 Department of Cytology and Histology, Saint Petersburg State University, \\ Universitetskaya nab. 7-9, Saint Petersburg, 199034, Russian Federation \\ Address correspondence and requests for materials to Elena Gaginskaya \\ elena.gaginskaya@spbu.ru
}

\begin{abstract}
Lampbrush chromosomes represent transcriptionally active chromosomes that exist during the extended diplotene phase in the growing ovarian eggs of many vertebrate animals, except therian mammals. They are excellent for studying chromosome structure and aspects of RNA transcription. Because of their giant size they allow a great increase in the resolution for physical gene mapping. Lampbrush chromosomes have no connection with the nuclear envelope, and can be manually dissected from living oocyte nuclei. Here we present the complete protocol for lampbrush chromosome microsurgical isolation from avian and reptilian germinal vesicles. These chromosome preparations can be further used for fluorescence in situ hybridization (FISH) and immunostaining.
\end{abstract}

Keywords: nucleus, germinal vesicle, lampbrush chromosomes, meiotic chromosomes, microsurgical isolation, Aves, Reptilia

\section{Introduction}

Volodkina, V., and Gaginskaya E. 2017. Preparation of lampbrush chromosomes dissected from avian and reptilian growing oocytes. Bio. Comm. 62(3): 165-168. https:// doi.org/10.21638/11701/spbu03.2017.302

Author's information: Alsu Saifitdinova, Ph.D., Director, orcid.org/0000-00021221-479X, Researcher ID: C-1104-2011; Svetlana Galkina, Ph.D., Researcher, orcid. org/0000-0002-7034-2466, Researcher ID: J-2493-2013; Valeriia Volodkina, student; Elena Gaginskaya, Dr. Sci., Professor, orcid. org/0000-0003-4967-9053, Researcher ID: C-9963-2013

Manuscript Editor: Prof. Em. Neil Jones, Institute of Biological, Environmental and Rural Sciences, Aberystwyth University, Aberystwyth, U. K.

Received: November 13, 2017;

Revised: November 22, 2017;

Accepted: November 25, 2017;

Copyright: (c) 2017 Saifitdinova et al. This is an open-access article distributed under the terms of the License Agreement with Saint Petersburg State University, which permits to the authors an unrestricted distribution and self-archiving free of charge.

Funding: The research was carried out under project 15-04-05684 financed by Russian Basic Research Foundation. V.V. was supported by a grant from the Saint Petersburg State University (1.42.961.2016)
Lampbrush chromosomes are chromosomes of a specific form which appear in growing oocyte nuclei (germinal vesicles) of many animals, including birds and reptiles, due to a highly intense transcription of a variety of sequences. They acquired this name in 1892 because of their extremely large size and a formal resemblance to the brush for cleaning kerosene lamp glass (Rückert 1892). The cause of such similarity lies in the high degree of chromatin despiralization and the presence of numerous lateral loops of DNA, which are extended from chromomeres along the chromosome axis and actively transcribed by RNA polymerase II (Callan 1986). The high degree of decondensation, combined with the preservation of chromosome axial structure, have made lampbrush chromosomes a unique tool for high-resolution gene mapping and genome assembly data verification (Diaz and Gall 1985; Ogawa et al., 1997; Andreozzi et al., 2001; Galkina et al., 2006; Solinhac et al., 2010; Zlotina et al., 2010; Bellott et al., 2017). The first attempts of manual microdissection of lampbrush chromosomes from avian oocyte nuclei were made in the 1960s (Koecke and Muller 1965; Gaginskaya and Gruzova 1969; Ahmad 1970). Although these attempts were based on the technique previously developed for amphibians (Gall 1954; Callan and Lloyd 1960; Macgregor and Varley, 1983), in the area of avian lampbrush chromosome manipulations, the team of the Laboratory of Chromosome Structure and Function of Saint Petersburg State University definitely takes world precedence (Macgregor, 2012). Systematic studies of lampbrush chromosomes from the nuclei of bird oocytes began after publications by Ekaterina Kropotova and Elena Gaginskaya (1984), and by Nancy Hutchison (Hutchinson 1987), where methods of lampbrush chromosome manipulations and preparation for both light and electron microscopy were adapted to the avian lampbrush chromosomes. Irina Solovei, Elena 
Gaginskaya, and Herbert Macgregor made a notable contribution to the development of this method (Solovei et al., $1992 ; 1993 ; 1994 ; 1995)$. Over the last years, however, the main protocol underwent some revisions and improvements aimed at obtaining high quality avian and reptilian lampbrush chromosome preparations that would be suitable for further analysis using immunocytochemistry and fluorescence in situ hybridization (FISH).

\section{Equipment and instruments}

- Stereo microscope with bottom lighting and variable intensity control, total magnification from $10 \mathrm{x}$ to 100x and min. focal length of $60 \mathrm{~mm}$.

- Cytological centrifuge with cooling, min. $3500 \mathrm{~g}$ acceleration, with a batch rotor and baskets with lids, allowing horizontal placement of $76 \times 24 \mathrm{~mm}$ preparation slides.

- $6 V$ DC power supply equipped with cleats.

- Stainless steel ophthalmic scissors.

- $115 \mathrm{~mm}$ histological forceps.

- Variable volume micropipettes (up to $20 \mu \mathrm{l}$ and up to $200 \mu \mathrm{l})$ with polypropylene sterile tips.

- Variable volume micropipettes (up to $10 \mu \mathrm{l}$ ) with tips made of transparent low-adhesion polypropylene or pipette-stripper with replaceable glass tips with a 150$200 \mu \mathrm{m}$ in diameter with rounded edges (optional).

- Glass micropipettes that can be made from sterile Pasteur glass pipettes by stretching the tip after heating in a burner flame (optional). Depending on the strength and speed of stretching, blanks of different length and thickness can be obtained. With pre-annealed forceps the blanks are broken precisely orthogonally at the level where the diameter of the glass tube is slightly larger than required. The tip of the pipette is placed into the burner flame for melting and smoothing. A small pipette bulb is placed on the wide end of the pipette.

- Tungsten needles are used for isolation of oocyte nucleus and removal of nuclear envelope and made of tungsten wire by electrolytic grinding. A wire of 120 500 microns in diameter is cut into $5-7 \mathrm{~cm}$ blanks. Then a platinum cathode is connected to the DC source $(6 \mathrm{~V})$ and immersed into a glass with freshly prepared $10 \% \mathrm{KOH}$ solution. Tungsten blanks are connected to the positive pole of the current source with an electric clamp and immersed into a beaker with electrolyte. In order to obtain the desired shape of the needle tip, the blank should be immersed into the solution gradually for smooth diameter reduction. As appropriate, the needles can be made of any desired shape (most often, straight, slightly curved and L-shaped tungsten needles are used). The tips of the needles are sharpened additionally by brief contact with the surface of electrolyte solution during operation. This process is controlled under a stereomicroscope. Such needles can be gripped in collet holders. Tungsten tools are sterilized in the flame of the burner.
- Transparent embryo dish with 1-2 ml cavity. Before use, the embryo dishes should be washed in soap solution and rinsed with distilled water.

- $\quad 76 \times 24 \mathrm{~mm}$ slides, thickness up to $1.2 \mathrm{~mm}$. The slides are washed in $7 \mathrm{X}$ detergent solution (7X or 7X-OMatic) followed by rinsing with plenty of distilled water. The slides are dried in a thermostat at $+60^{\circ} \mathrm{C}$.

- $22 \times 22 \mathrm{~mm}$ borosilicate glass chambers, $1.2 \mathrm{~mm}$ thick, with 1 to $4.4 \mathrm{~mm}$ holes. The chambers are pretreated in $70 \%$ ethanol and wiped dry before use. The chambers are fixed onto the slide plates strictly in the center with natural rubber glue and left to dry for at least 16 hours at room temperature. The prepared chambers can be stored at $+4^{\circ} \mathrm{C}$. The glue layer quality is inspected immediately before use. Excess glue is removed from chamber holes, and holes are filled with hypotonic solution for chromosome isolation.

- Glass Petri dishes for preserving the chamber slides from drying during chromosome spreading at $+4^{\circ} \mathrm{C}$. Dry filter paper is placed at the bottom of the dish to prevent condensate.

- A glass container with a ground stopper for ovary storage and prevention from dehydration during the work process. A sheet of filter paper impregnated with buffer solution is placed at the bottom of the container for this purpose.

- Saline solution coolant in a plastic bag, min. size $15 \times 15 \mathrm{~cm}$. The bag with the coolant should be pre-frozen at $-20^{\circ} \mathrm{C}$ and covered with paper tissue during the work process to prevent the preparation slides from freezing to it.

- $\quad 75 \mathrm{~cm}$ tall $50 \mathrm{ml}$ glass beakers and disposable plastic dishes for solution storage and fixation of the prepared slides.

- Diamond pencil for marking slides.

\section{Solutions}

- Medium for nucleus isolation «5:1»: $83 \mathrm{mM} \mathrm{KCl}$, $17 \mathrm{mM} \mathrm{NaCl}, 6.5 \mathrm{mM} \mathrm{Na}_{2} \mathrm{HPO}_{4}, 3.5 \mathrm{mM} \mathrm{NaH}_{2} \mathrm{PO}_{4}$ $\left(\mathrm{KH}_{2} \mathrm{PO}_{4}\right), \mathrm{pH} 7,0$.

- Hypotonic medium for chromosome isolation «1:4»: «5:1» medium diluted 4 times and containing additionally $0.25 \mathrm{mM} \mathrm{MgCl}_{2}, 0.1 \%$ formaldehyde.

- Fixative solution: «1:4» medium with addition of $2 \%$ formaldehyde. For immunocytochemistry add $0.25 \mathrm{mM} \mathrm{CaCl} 2 ; 0.25 \mathrm{mM} \mathrm{MgCl} 2$. PBS (phosphate buffered saline) with $2 \%$ formaldehyde also can be used.

- Fresh ethanol solutions for slide dehydration: $50 \%$, $70 \%$ and $96 \%$.

\section{Protocol}

All manipulations related to lampbrush chromosome isolation should be conducted without heating the solutions and slides, empty embryo dishes and chamber slides must constantly be kept on a cooling surface. The glass contain- 


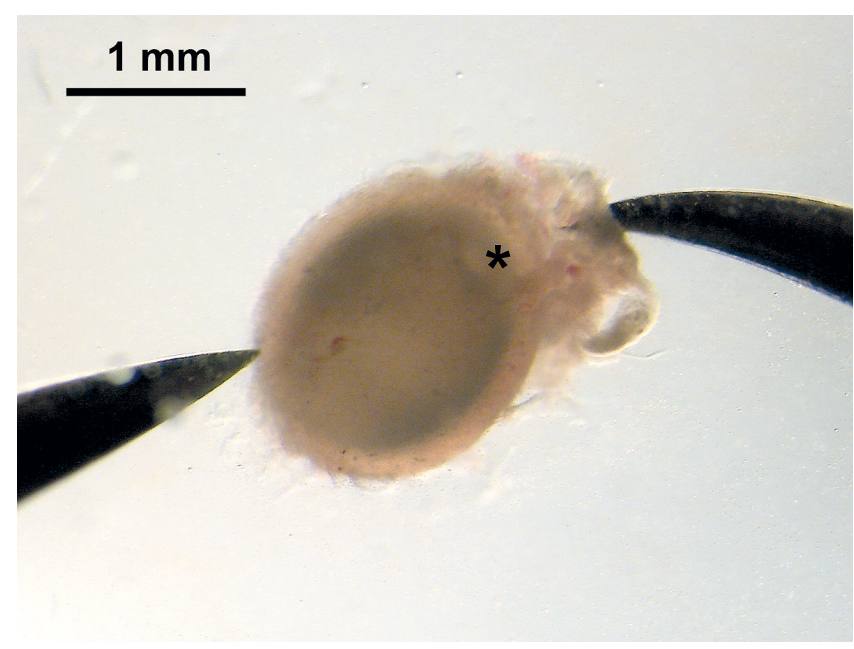

Fig. 1. Cutting an oocyte at the stage of intense growth out of a chicken ovary. Its diameter will be nearly $2 \mathrm{~mm}$, including the tightly fitting follicular epithelium envelope. The area of nucleus location is marked with an asterisk. Hold the oocyte with one needle while the first dissection is being made with the other needle. Then use it to widen the opening and release the content from the follicular membrane

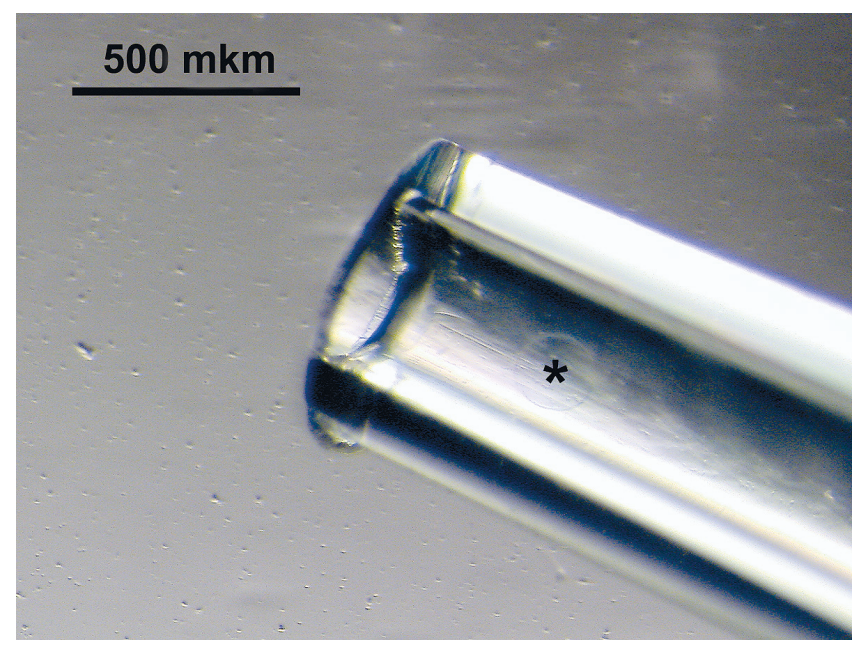

Fig. 3. Oocyte nucleus cleared of excess ooplasm (marked with an asterisk) during transfer into a prepared chamber slide

er with recently dissected ovary and preparations must be kept in the fridge at $+4^{\circ} \mathrm{C}$ during spreading. All oocytes in the ovary are surrounded by follicular epithelium and located in the cortical layer of the ovary, the largest of them protruding from its surface. Oocytes of $0.3-1.5 \mathrm{~mm}$ in diameter are cut out of the ovary with scissors and put into an embryo dish containing chilled « $5: 1 »$ medium. The position of the nucleus can be identified under a stereomicroscope as a bright area inside the oocyte. Then the oocyte should be arranged in a manner preventing nucleus damage during follicle dissection (Fig. 1).

With two sharp tungsten needles, tear the follicular cover and the plasma membrane of the oocyte and release the nucleus from the ooplasm (Fig. 2). The intact nucleus is completely transparent, yet clearly visible due to the opal-

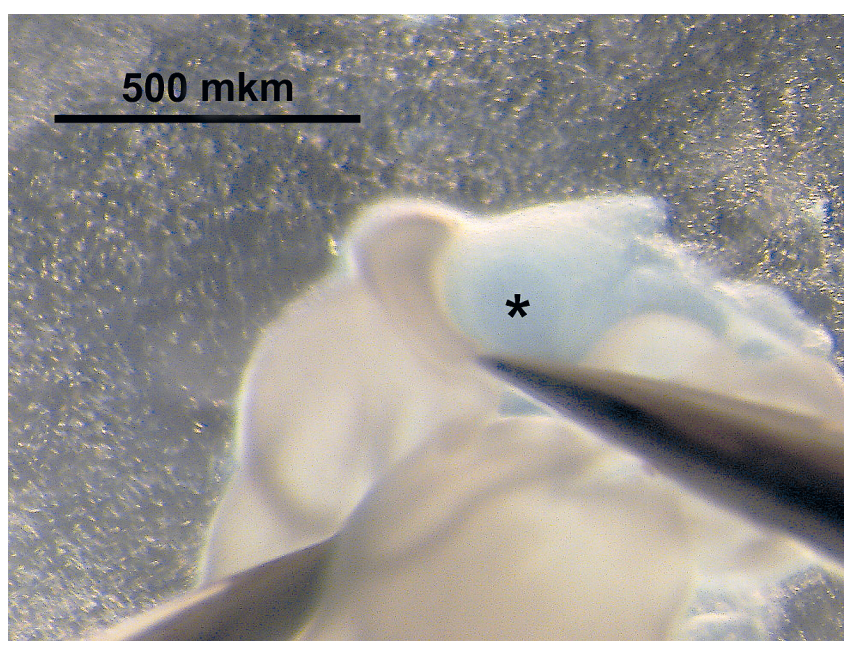

Fig. 2. Chicken oocyte content with a nucleus in it (marked with asterisk). Carefully remove any excess ooplasm (yolk) with the aid of needles and gently release the core. While doing it, avoid needle tip contact with the nuclear surface

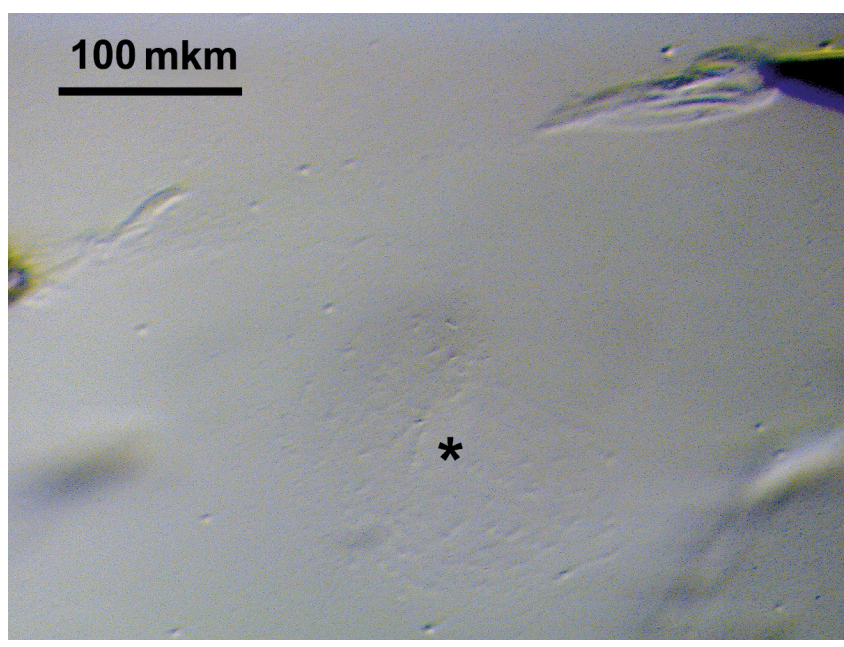

Fig. 4. The nuclear envelope being removed with the aid of thin needles. Karyoplasm with chromosomes sediments to the bottom of the chamber (marked with an asterisk)

escent nuclear envelope, and has a regular spherical shape. Transfer the nucleus into another embryo dish containing hypotonic «1:4» medium, and try to remove any ooplasm particles by pipetting the nucleus in and out. Then transfer the nucleus with the same pipette into a chamber slide filled with «1:4» medium (Fig. 3). Using thin tungsten needles, remove the nuclear envelope and leave the chromosomes to spread at the bottom of the chamber under the effect of hypotonic solution and gravitational force (Fig. 4).

Keep the chambers containing dissected oocyte nucleus in a Petri dish at $+4^{\circ} \mathrm{C}$ for 30 minutes, then centrifuge them for 30 minutes at $3500 \mathrm{~g}$ in a cold $\left(+4^{\circ} \mathrm{C}\right)$ cytological centrifuge. Fix chromosome slides in $2 \%$ formaldehyde solution for 30 minutes at room temperature. Dehydrate the preparations in $50 \%$ and $70 \%$ ethanol for 5 minutes 
per solution, and then in another portion of $70 \%$ ethanol overnight. The next day, mark the positions of the chamber holes with a diamond pencil on the bottom surface of the slide. Peel the chambers off the slide with the aid of a razor blade, transfer the chromosome preparations into $96 \%$ ethanol for 5 minutes and then air-dry. Dried slides can be stored at room temperature for several months. When preparing lampbrush chromosomes for further immunocytochemical studies, add bivalent metal salts $(0.25 \mathrm{mM}$ $\mathrm{CaCl}_{2}, 0.25 \mathrm{mM} \mathrm{MgCl}_{2}$ ) to «1:4» medium and fixative for preserving the chromatin ribonucleoprotein complexes. In this case, the preparations should be kept in $70 \%$ ethanol at $+4^{\circ} \mathrm{C}$ without drying and used within a week.

\section{Conclusion}

Lampbrush chromosomes from growing oocytes represent a good model system for the analysis of chromosome structure, transcription and processing events with the highest resolution. Nevertheless, contrary to amphibian oocytes the lampbrush chromosome dissection from avian germinal vesicles has been found to have certain difficulties. These difficulties are caused by the follicular envelope, which we are unable to remove without damaging the oocyte membrane because of numerous protrusions of follicular cells into the ooplasm (transosome formation). As a result, it is impossible to conduct a prolonged incubation of living oocyte. It is now possible to find a way to overcome this problem by developing methods of follicular epithelium removal and solutions for released oocyte maintenance.

\section{Acknowledgements}

The authors are grateful to Anton Radaev, Mikhail Kuleshin for technical support and Alissa Gousseva for manuscript editing. All manipulations were performed using the equipment of Chromas Core Facility of Saint Petersburg State University Research Park.

\section{References}

Ahmad, M. S. 1970. Development, structure and composition of lampbrush chromosomes in domestic fowl. Canadian Journal of Genetics and Cytology 12: 728-737. https://doi. org/10.1139/g70-095

Andreozzi, L., Federico, C., Motta, S., Saccone, S., Sazanova, A. L., Sazanov, A. A., Smirnov, A. F., Galkina, S. A., Lukina, N. A., and Rodionov, A. V. 2001. Compositional mapping of chicken chromosomes and identification of the gene-richest regions. Chromosome Research 9: 521-532. https://doi.org/10.1023/A:1012436900788

Bellott, D.W., Skaletsky, H., Cho, T.J., Brown, L., Locke, D., Chen, N., Galkina, S., Pyntikova, T., Koutseva, N., Graves, T., Kremitzki, C., Warren, W. C., Clark, A. G., Gaginskaya, E. R., Wilson, R. K., Page, D. C. 2017. Avian $\mathrm{W}$ and mammalian $\mathrm{Y}$ chromosomes convergently retained dosage-sensitive regulators. Nature Genetics 49: 387-394. https://doi.org/10.1038/ng.3778

Callan, H. G. 1986 Lampbrush Chromosomes. Berlin, Heidelberg: Springer-Verlag.

Callan, H. G., and Lloyd, L. 1960. Lampbrush chromosomes of crested newts Triturus cristatus (Laurenti). Philosophical
Transactions of the Royal Society of London, Series B 243: 135-219. https://doi.org/10.1098/rstb.1960.0007

Diaz, M. O. and Gall, J. G. 1985. Giant readthrough transcription units at the histone loci on lampbrush chromosomes of the newt Notophthalmus. Chromosoma 92: 243-253. https://doi.org/10.1007/BF00329807.

Gaginskaya, E. R. and Gruzova, M. N. 1969. Peculiarities of the oogenesis in chaffinch. Tsitologiia 11: 1241-1251.

Galkina, S., Derjusheva, S., Fillon, V., Vignal, A., Crooijmans, R., Groenen, M., Rodionov, A. and Gaginskaya, E. 2006. FISH on avian lampbrush chromosomes produces higher resolution gene mapping. Genetica 128: 241-251. https:// doi.org/10.1007/s10709-005-5776-7.

Gall, J. G. 1954. Lampbrush chromosomes from oocyte nuclei of the newt. Journal of Morphology 94: 283-352. https:// doi.org/10.1002/jmor.1050940203.

Hutchison, N. 1987. Lampbrush chromosomes of the chicken, Gallus domesticus. Journal of Cell Biology 105: 1493-1500. https://doi.org/10.1083/jcb.105.4.1493.

Koecke, H. U., and Muller, M. 1965. Formwechel und Anzahl der Chromosomen bei Huhn und Ente. Naturwissenshchaften 52: 483. https://doi.org/10.1007/BF00626256.

Kropotova E. V., and Gaginskaya E. R. 1984. Hromosomy typa lampovyh schetok iz oocytov japonskogo perepela. Dannye svetovoi i elektronnoi mikroskopii [Lampbrush chromosomes from the Japanese quail oocytes. Optical light and electron microscopy data]. Tsitologia 26: 1008-1015.

Macgregor, H. C. 2012. Chromomeres revisited. Chromosome Research 20: 911-924. https://doi.org/10.1007/s10577012-9310-3.

Macgregor, H. C., and Varley, J. M. 1988. Working with animal chromosomes (2nd ed.). Chichester: John Wiley.

Ogawa, A., Solovei, I., Hutchison, N., Saitoh, Y., Ikeda, J-E., Macgregor, H. and Mizuno, S. 1997. Molecular characterization and cytological mapping of a non-repetitive DNA sequence region from the $\mathrm{W}$ chromosome of chicken and its use as a universal probe for sexing Carinatae birds. Chromosome Research 5: 93-101. https://doi. org/10.1023/A:1018461906913.

Rückert, J. 1892. Zur Entwicklungsgeschichte des Ovarialeies bei Selachiern. Anatomischer Anzeiger 7: 107-158.

Solinhac, R., Leroux, S., Galkina S, Chazara, O., Feve, K., Vignoles, F., Morisson, M., Derjusheva, S., Bed'hom, B., Vignal, A., Fillon, V. and Pitel, F. 2010. Integrative mapping analysis of chicken microchromosome 16 organization. BMC Genomics. 11: 616. https://dx.doi.org/10.1186/14712164-11-616.

Solovei, I., Gaginskaya, E., Allen, T., and Macgregor, H. 1992. A novel structure associated with a lampbrush chromosome in the chicken, Gallus domesticus. Journal of Cell Science 101: 759-772.

Solovei, I., Gaginskaya, E., Hutchinson, N., and Macgregor, H. 1993. Avian sex chromosomes in the lampbrush form: the ZW lampbrush bivalent from six species of bird. Chromosome Research 1: 153-166. https://doi.org/10.1007/BF00710769.

Solovei, I., Gaginskaya, E. R., and Macgregor, H. C. 1994. The arrangement and transcription of telomere DNA sequences at the ends of lampbrush chromosomes of birds. Chromosome Research 2: 460-470. https://doi. org/10.1007/BF01552869.

Solovei, I., Macgregor, H., and Gaginskaya, E. 1995. Single stranded nucleic acid binding structures on chicken lampbrush chromosomes. Journal of Cell Science 108: 1391-1396.

Zlotina, A., Galkina, S., Krasikova, A., Crooijmans, R.P. M. A., Groenen, M.A. M., Gaginskaya, E. and Derjusheva, S. 2010. Precise centromere positioning on chicken chromosome 3. Cytogenetics and Genome Research 129: 310313. https://doi.org/10.1159/000314923. 